\title{
Convenient Stability Criteria for Difference Approximations of Hyperbolic Initial-Boundary Value Problems. II
}

\author{
By Moshe Goldberg* and Eitan Tadmor**
}

\begin{abstract}
The purpose of this paper is to extend the results of [4] in order to achieve more versatile, convenient stability criteria for a wide class of finite-difference approximations to initial-boundary value problems associated with the hyperbolic system $\mathbf{u}_{t}=A \mathbf{u}_{x}+B \mathbf{u}+\mathbf{f}$ in the quarter plane $x \geqslant 0, t \geqslant 0$. With these criteria, stability is easily established for a large number of examples, where many of the cases studied in the recent literature are included and generalized.
\end{abstract}

0. Introduction. In this paper we sharpen and extend the results of [4] in order to achieve more versatile, convenient, sufficient stability criteria for a large class of approximations to the initial-boundary value problem associated with the hyperbolic system $\mathbf{u}_{t}=A \mathbf{u}_{x}+B \mathbf{u}+\mathbf{f}$ in the quarter plane $x \geqslant 0, t \geqslant 0$. Our difference approximation consists of a general difference scheme-explicit or implicit, dissipative or not, two-level or multi-level-and boundary conditions of a wider type than discussed in [4].

As in [4], we restrict attention to the case where the outflow components of the principal part of the boundary conditions are translatory, i.e., determined at all boundary points by the same coefficients. Such boundary conditions are commonly used in practice; and in particular, when the boundary consists of a single point, the boundary conditions are translatory by definition.

Throughout the paper we assume that the basic scheme is stable for the pure Cauchy problem, and that the other assumptions which guarantee the validity of the Gustafsson-Kreiss-Sundström stability theory in [5] hold for our case. We then raise the question of stability for our approximation in the sense of Definition 3.3 in [5].

Our stability analysis begins in Section 2, where we show (Theorem 2.1) that our entire approximation is stable if and only if the scalar outflow components of its principal part are stable. Thus, our global stability question is reduced to that of a

Received March 13, 1986.

1980 Mathematics Subject Classification. Primary 65M10; Secondary 65N10.

* Research sponsored in part by the Air Force Office of Scientific Research, United States Air Force Grant AFOSR-83-0150. Part of this research was carried out while the author visited the University of California in Los Angeles and Santa Barbara, and California Institute of Technology.

** Research supported in part by NASA Contract NAS1-17070 while the author was in residence at ICASE, NASA Langley Research Center, Hampton, VA 23665. Additional support was provided by NSF Grant DMS85-03294 and ARO Grant DAAG29-85-K-0190 while in residence at UCLA. The author is a Bat-Sheva Foundation Fellow. 
scalar, homogeneous, outflow problem which, as in [4], is the main subject of this paper.

We state our stability criteria for the reduced problem in Theorems 3.1 and 3.2 of Section 3. These criteria depend both on the basic scheme and the boundary conditions, but very little on the intricate interaction between the two. It follows that our criteria provide in many cases a convenient, easily-to-check alternative to the well-known Gustafsson-Kreiss-Sundström criterion in [5].

We proceed in Section 3 to use our stability criteria in Theorems 3.1 and 3.2 together with Lemmas 3.1 and 3.2 in order to establish all our previous examples in [4] as well as new ones. This includes a host of dissipative and nondissipative examples that include and generalize many of the cases studied in the recent literature; e.g., [1]-[10], [12]-[15].

As in [4], we point out that there is no difficulty in extending our stability criteria to two-boundary problems, since if the corresponding left and right quarter-plane problems are stable then, by Theorem 5.4 of [5], the original problem is stable as well.

We also remark that there are no essential obstacles in extending our results to initial-boundary value problems with variable coefficients.

1. The Differential Problem and the Difference Approximation. Consider the first-order hyperbolic system of partial differential equations

(1.1a) $\partial \mathbf{u}(x, t) / \partial t=A \partial \mathbf{u}(x, t) / \partial x+B \mathbf{u}(x, t)=\mathbf{f}(x, t), \quad x \geqslant 0, t \geqslant 0$,

where $\mathbf{u}(x, t)=\left(u^{(1)}(x, t), \ldots, u^{(n)}(x, t)\right)^{\prime}$ is the unknown vector (prime denoting the transpose $), \mathbf{f}(x, t)=\left(f^{(1)}(x, t), \ldots, f^{(n)}(x, t)\right)^{\prime}$ a given $n$-vector, and $A$ and $B$ fixed $n \times n$ matrices such that $A$ is symmetric and nonsingular. Without restriction we may assume that the system is given in characteristic variables, namely $A$ is diagonal of the form

$$
A=\left(\begin{array}{cc}
A^{\mathrm{I}} & 0 \\
0 & A^{\mathrm{II}}
\end{array}\right), \quad A^{\mathrm{I}}>0, A^{\mathrm{II}}<0,
$$

where $A^{\mathrm{I}}$ and $A^{\mathrm{II}}$ are of orders $l \times l$ and $(n-l) \times(n-l)$, respectively.

The solution of $(1.1 \mathrm{a})$ is uniquely determined if we prescribe initial values

$$
\mathbf{u}(x, 0)=\stackrel{\mathrm{u}}{ }(x), \quad x \geqslant 0,
$$

and boundary conditions

$$
\mathbf{u}^{\mathrm{II}}(0, t)=S \mathbf{u}^{\mathrm{I}}(0, t)+\mathbf{g}(t), \quad t \geqslant 0,
$$

where $S$ is a fixed $(n-l) \times l$ coupling matrix, $\mathbf{g}(t)$ a given $(n-l)$-vector, and

$$
\mathbf{u}^{\mathrm{I}}=\left(u^{(1)}, \ldots, u^{(l)}\right)^{\prime}, \quad \mathbf{u}^{\mathrm{II}}=\left(u^{(l+1)}, \ldots, u^{(n)}\right)^{\prime}
$$

a partition of $\mathbf{u}$ into its outflow and inflow components, respectively, corresponding to the partition of $A$ in (1.2).

Introducing a mesh size $\Delta x>0, \Delta t>0$, such that $\lambda \equiv \Delta t / \Delta x=$ constant, and using the notation $\mathbf{v}_{\nu}(t)=\mathrm{v}(\nu \Delta x, t)$, we approximate (1.1a) by a general, consistent, 
two-sided, solvable basic scheme of the form

$$
\begin{gathered}
Q_{-1} \mathbf{v}_{\nu}(t+\Delta t)=\sum_{\sigma=0}^{s} Q_{\sigma} \mathbf{v}_{\nu}(t-\sigma \Delta t)+\Delta t \mathbf{b}_{\nu}(t), \quad \nu=r, r+1, \ldots, \\
Q_{\sigma}=\sum_{j=-r}^{p} A_{j \sigma} E^{j}, \quad E \mathbf{v}_{\nu}=\mathbf{v}_{\nu+1}, \quad \sigma=-1, \ldots, s,
\end{gathered}
$$

where the $n \times n$ coefficient matrices $A_{j \sigma}$ are polynomials in $\lambda A$ and $\Delta t B$, and the $n$-vectors $\mathbf{b}_{\nu}(t)$ depend on $\mathbf{f}(x, t)$ and its derivatives.

The difference equations in (1.4) have a unique solution $\mathbf{v}_{\nu}(t+\Delta t)$ if we provide initial values

$$
\mathbf{v}_{\nu}(\mu \Delta t)=\stackrel{\mathrm{v}}{\nu}_{\nu}(\mu \Delta t), \quad \mu=0, \ldots, s, \nu=0,1,2, \ldots,
$$

and specify, at each time level $t=\mu \Delta t, \mu=s, s+1, \ldots$, boundary values $\mathbf{v}_{\nu}(t+\Delta t)$, $\nu=0, \ldots, r-1$. These boundary values will be determined by boundary conditions of the form

$$
\begin{gathered}
T_{-1}^{(\nu)} \mathbf{v}_{\nu}(t+\Delta t)=\sum_{\sigma=0}^{q} T_{\sigma}^{(\nu)} \mathbf{v}_{\nu}(t-\sigma \Delta t)+\Delta t \mathbf{d}_{\nu}(t), \quad \nu=0, \ldots, r-1, \\
T_{\sigma}^{(\nu)}=\sum_{j=0}^{m} C_{j \sigma}^{(\nu)} E^{j}, \quad \sigma=-1, \ldots, q
\end{gathered}
$$

where the $n \times n$ matrices $C_{j \sigma}^{(\nu)}$ depend on $A, \Delta t B$ and $S$, and the $n$-vectors $\mathbf{d}_{\nu}(t)$ are functions of $\mathbf{f}(x, t), \mathbf{g}(t)$ and their derivatives.

We shall assume that the leading coefficients $C_{0(-1)}^{(\nu)}$ are nonsingular, thus assuring that the boundary conditions (1.6a) can be solved for the required boundary values $\mathbf{v}_{\nu}(t+\Delta t), \nu=r-1, \ldots, 0$, in terms of neighboring values of $\mathbf{v}_{\nu}$.

We shall further assume that the matrices $C_{j \sigma}^{(\nu)}$ depend weakly on $B$, in that $B$ introduces a mere $O(\Delta t)$ perturbation in these matrices. This assumption holds for all practical boundary conditions where the elements of $C_{j \sigma}^{(\nu)}$ are polynomials in the entries of $\Delta t B$.

Finally, we assume that in accordance with the partition of $A$ in (1.2), the $C_{j \sigma}^{(\nu)}$ can be written as

$$
C_{j \sigma}^{(\nu)}=\left(\begin{array}{cc}
C_{j \sigma}^{\mathrm{II}} & C_{j \sigma}^{\mathrm{III}(\nu)} \\
C_{j \sigma}^{\mathrm{II} \mathrm{I}(\nu)} & C_{j \sigma}^{\mathrm{IIII}(\nu)}
\end{array}\right),
$$

where

$$
\text { the } C_{j \sigma}^{\mathrm{II}} \text { are independent of } \nu \text {, }
$$$$
\text { the } C_{j \sigma}^{\mathrm{II}} \text { are diagonal when } B=0 \text {, }
$$$$
\text { the } C_{j \sigma}^{\mathrm{III}(\nu)}=0 \text { when } B=0 \text {, }
$$

and

$$
C_{j \sigma}^{\mathrm{IIII}(\nu)}=0 \text { for } j>0 \text { and } \sigma>-1 \text { when } B=0
$$


Let us now set

$$
\mathbf{v}_{\nu}=\left(\begin{array}{c}
\mathbf{v}_{\nu}^{\mathrm{I}} \\
\mathbf{v}_{\nu}^{\mathrm{II}}
\end{array}\right), \quad \mathbf{d}_{\nu}=\left(\begin{array}{c}
\mathbf{d}_{\nu}^{\mathrm{I}} \\
\mathbf{d}_{\nu}^{\mathrm{II}}
\end{array}\right),
$$

according to the partition of $\mathbf{u}$ in (1.3); the boundary conditions (1.6a, b) then split into two groups:

$$
\begin{aligned}
& T_{-1}^{\mathrm{II}} \mathbf{v}_{\nu}^{\mathrm{I}}(t+\Delta t)+T_{-1}^{\mathrm{III}(\nu)} \mathbf{v}_{\nu}^{\mathrm{II}}(t+\Delta t) \\
& =\sum_{\sigma=0}^{q}\left[T_{\sigma}^{\mathrm{II}} \mathbf{v}_{\nu}^{\mathrm{I}}(t-\sigma \Delta t)+T_{\sigma}^{\mathrm{III}(\nu)} \mathbf{v}_{\nu}^{\mathrm{II}}(t-\sigma \Delta t)\right]+\Delta t \mathbf{d}_{\nu}^{\mathrm{I}}(t), \\
& T_{\sigma}^{\mathrm{II}}=\sum_{j=0}^{m} C_{j \sigma}^{\mathrm{II}} E^{j}, \quad T_{\sigma}^{\mathrm{III}(\nu)}=\sum_{j=0}^{m} C_{j \sigma}^{\mathrm{III}(\nu)} E^{j}, \\
& \quad \sigma=-1, \ldots, q, \nu=0, \ldots, r-1 ;
\end{aligned}
$$

and

$$
\begin{aligned}
T_{-1}^{\mathrm{II} I(\nu)} \mathbf{v}_{\nu}^{\mathrm{I}}(t+\Delta t)+T_{-1}^{\mathrm{II} \mathrm{II}(\nu)} \mathbf{v}_{\nu}^{\mathrm{II}}(t+\Delta t) \\
=\sum_{\sigma=0}^{q}\left[T_{\sigma}^{\mathrm{II}(\nu)} \mathbf{v}_{\nu}^{\mathrm{I}}(t-\sigma \Delta t)+T_{\sigma}^{\mathrm{II} \mathrm{II}(\nu)} \mathbf{v}_{\nu}^{\mathrm{II}}(t-\sigma \Delta t)\right]+\Delta t \mathbf{d}_{\nu}^{\mathrm{II}}(t), \\
T_{\sigma}^{\mathrm{II} \alpha(\nu)}=\sum_{j=0}^{m} C_{j \sigma}^{\mathrm{II} \alpha(\nu)} E^{j}, \quad \alpha=\mathrm{I}, \mathrm{II}, \sigma=-1, \ldots, q, \nu=0, \ldots, r-1,
\end{aligned}
$$

which we refer to as the outflow and inflow boundary conditions, respectively. With this formulation it is a simple matter to verify that the boundary conditions in our previous papers [3], [4] constitute a special case of the present ones. Hence, the argument concluding Section 1 of [3] implies that boundary conditions of the form (1.6) can be constructed to any degree of accuracy.

It should be pointed out that the outflow boundary conditions in (1.7b) are quite general, despite the apparent restrictions in (1.6c)-(1.6e). Indeed, (1.6c) is not much of a restriction since in practice the outflow boundary conditions are often translatory, i.e., determined at all boundary points by the same coefficients. In particular, if the numerical boundary consists of a single point, then the boundary conditions are translatory by definition, so (1.6c) holds automatically. The restrictions in (1.6d), (1.6e) pose no great difficulties either, since they are satisfied by all reasonable boundary conditions, where for $B=0$ the $C_{j \sigma}^{\mathrm{II}}$ usually reduce to polynomials in the diagonal block $A^{\mathrm{I}}$, and the $C_{j \sigma}^{\mathrm{I} \mathrm{II}(\nu)}$ vanish.

We realize that in view of the restriction in (1.6f) the inflow boundary conditions (1.7c) are not quite as general as the outflow ones. If, however, the boundary consists of a single point, then such conditions can be achieved in a trivial manner, simply by duplicating the analytic condition (1.1c), which gives

$$
\mathbf{v}_{0}^{\mathrm{II}}(t+\Delta t)=S \mathbf{v}_{0}^{\mathrm{I}}(t+\Delta t)+\mathbf{g}(t+\Delta t) .
$$

2. The Reduced Approximation. The difference approximation is now completely defined by (1.4)-(1.6), and we wish to apply to it the stability theory of Gustafsson, Kreiss and Sundström in [5]. For this purpose we assume from now on that the basic scheme $(1.4 \mathrm{a})$ is stable for the pure Cauchy problem $(-\infty<\nu<\infty)$ and that the 
other assumptions in [5] are satisfied as well. With this, the Gustafsson-KreissSundström theory holds for our case*** and we ask whether approximation (1.4)-(1.6) is stable in the sense of Definition 3.3 of [5].

In Theorem 2.1, which concludes this section, we shall reduce the above stability question to that of a scalar outflow approximation with homogeneous boundary conditions. In order to obtain this reduction, we begin, as in [3], by recalling Lemma 10.3 of [5] which provides a necessary and sufficient determinantal stability criterion stated entirely in terms of the homogeneous part of the difference approximation. This criterion immediately implies that for stability purposes we may consider (1.4)-(1.6) with

$$
\mathbf{b}_{\nu}(t)=\mathbf{d}_{\nu}(t)=0 \text {. }
$$

Moreover, since $B$ introduces a mere $O(\Delta t)$ perturbation in the matrix coefficients $A_{j \sigma}$ and $C_{j \sigma}^{(\nu)}$, then by Theorem 4.3 of [5] our approximation is stable if and only if it is stable for $B=0$. Setting $B=0$, we get (1.6d)-(1.6f), which together with (2.1) proves that our stability problem is equivalent to that of the principal part of (1.4)-(1.6), given by a basic scheme of the form

$$
\begin{gathered}
Q_{-1} \mathbf{v}_{\nu}(t+\Delta t)=\sum_{\sigma=0}^{s} Q_{\sigma} \mathbf{v}_{\nu}(t-\sigma \Delta t), \quad \nu=r, r+1, \ldots, \\
Q_{\sigma}=\sum_{j=-r}^{p} A_{j \sigma} E^{j}, \quad \sigma=-1, \ldots, s ;
\end{gathered}
$$

with initial values

$$
\mathbf{v}_{\nu}(\mu \Delta t)=\stackrel{\circ}{v}_{\nu}(\mu \Delta t), \quad \mu=0, \ldots, s, \nu=0,1,2, \ldots,
$$

and outflow and inflow boundary conditions described, respectively, by

$$
\begin{aligned}
T_{-1}^{\mathrm{II}} \mathbf{v}_{\nu}^{\mathrm{I}}(t+\Delta t) & =\sum_{\sigma=0}^{q} T_{\sigma}^{\mathrm{II}} \mathbf{v}_{\nu}^{\mathrm{I}}(t-\sigma \Delta t), \quad \nu=0, \ldots, r-1, \\
T_{\sigma}^{\mathrm{II}} & =\sum_{j=0}^{m} C_{j \sigma}^{\mathrm{II}} E^{j}, \quad \sigma=-1, \ldots, q,
\end{aligned}
$$

$$
C_{0(-1)}^{\mathrm{II}} \text { nonsingular, } \quad C_{j \sigma}^{\mathrm{II}} \text { diagonal and independent of } \nu,
$$

and

$$
\begin{aligned}
& C_{0(-1)}^{\mathrm{II} \mathrm{II}(\nu)} \mathbf{v}_{\nu}^{\mathrm{II}}(t+\Delta t) \\
& \quad=\sum_{\sigma=0}^{q} T_{\sigma}^{\mathrm{II} \mathrm{I}(\nu)} \mathbf{v}_{\nu}^{\mathrm{I}}(t-\sigma \Delta t)-T_{-1}^{\mathrm{II} \mathrm{I}(\nu)} \mathbf{v}_{\nu}^{\mathrm{I}}(t+\Delta t), \quad \nu=0, \ldots, r-1, \\
& T_{\sigma}^{\mathrm{II} \mathrm{I}(\nu)}=\sum_{j=0}^{m} C_{j \sigma}^{\mathrm{II} \mathrm{I}(\nu)} E^{j}, \quad \sigma=-1, \ldots, q, \\
& C_{0(-1)}^{\mathrm{II} \mathrm{II}(\nu)} \text { nonsingular, } \quad \nu=0, \ldots, r-1 .
\end{aligned}
$$

This yields:

Lemma 2.1 (compare [3, Lemma 2.1]). Approximation (1.4)-(1.6) is stable if and only if its principal part in (2.2) is stable.

\footnotetext{
in [3].

*** The applicability of [5] to our approximation is further discussed in the first paragraph of Section 2
} 
Since now $B=0$ and $\mathbf{b}_{\nu}(t)=0$, then, evidently, the basic scheme (2.2a) is consistent with the principal part of (1.1a), i.e., with the homogeneous system

$$
\partial \mathbf{u}(x, t) / \partial t=A \partial \mathbf{u}(x, t) / \partial x .
$$

Furthermore, $B=0$ implies that the coefficients $A_{j \sigma}$ of the basic scheme (2.2a) are now polynomials only in $\lambda A$. Thus, the $A_{j \sigma}$ are diagonal matrices; and putting

$$
A_{j \sigma}=\left(\begin{array}{cc}
A_{j \sigma}^{\mathrm{I}} & 0 \\
0 & A_{j \sigma}^{\mathrm{II}}
\end{array}\right)
$$

in accordance with the partition of $A$ in (1.2), we may split the basic scheme (2.2a) and the initial values $(2.2 \mathrm{~b})$ into two parts:

$$
\begin{gathered}
Q_{-1}^{\mathrm{I}} \mathbf{v}_{\nu}^{\mathrm{I}}(t+\Delta t)=\sum_{\sigma=0}^{s} Q_{\sigma}^{\mathrm{I}} \mathbf{v}_{\nu}^{\mathrm{I}}(t-\sigma \Delta t), \quad \nu=r, r+1, \ldots, \\
Q_{\sigma}^{\mathrm{I}}=\sum_{j=-r}^{p} A_{j \sigma}^{\mathrm{I}} E^{j}, \quad \sigma=-1, \ldots, s, \\
\mathbf{v}_{\nu}^{\mathrm{I}}(\mu \Delta t)=\stackrel{\circ}{\nu}^{\mathrm{I}}(\mu \Delta t), \quad \mu=0, \ldots, s, \nu=0,1,2, \ldots ;
\end{gathered}
$$

and

$$
\begin{gathered}
Q_{-1}^{\mathrm{II}} \mathbf{v}_{\nu}^{\mathrm{II}}(t+\Delta t)=\sum_{\sigma=0}^{s} Q_{\sigma}^{\mathrm{II}} \mathbf{v}_{\nu}^{\mathrm{II}}(t-\sigma \Delta t), \quad \nu=r, r+1, \ldots, \\
Q_{\sigma}^{\mathrm{II}}=\sum_{j=-r}^{p} A_{j \sigma}^{\mathrm{II}} E^{j}, \quad \sigma=-1, \ldots, s, \\
\mathbf{v}_{\nu}^{\mathrm{II}}(\mu \Delta t)=\stackrel{\circ}{v}_{\nu}^{\mathrm{II}}(\mu \Delta t), \quad \mu=0, \ldots, s, \nu=0,1,2, \ldots
\end{gathered}
$$

We therefore view approximation (2.2) as made of outflow and inflow parts given by (2.4), (2.2c) and (2.5), (2.2d), respectively. And clearly, (2.2) is stable if and only if both parts are.

We observe the the outflow approximation (2.4), (2.2c) is self-contained, so we can solve it to obtain the outflow values

$$
\mathbf{v}_{\nu}^{\mathrm{I}}(t+\Delta t), \quad \nu=0,1,2, \ldots
$$

But now, these outflow values determine via $(2.2 \mathrm{~d})$ the required inflow boundary values

$$
\mathbf{v}_{\nu}^{\mathrm{II}}(t+\Delta t), \quad \nu=0, \ldots, r-1 .
$$

Hence, the stability question for approximation $(2.5),(2.2 \mathrm{~d})$ is equivalent to that of the inflow basic scheme (2.5a) with arbitrary inhomogeneous boundary values. Therefore, the above-mentioned Lemma 10.3 of [5] implies that, without affecting stability, we may replace these arbitrary inhomogeneous values by homogeneous ones:

$$
\mathbf{v}_{\nu}^{\mathrm{II}}(t+\Delta t)=0, \quad \nu=0, \ldots, r-1 .
$$

This gives us the new, self-contained inflow approximation (2.5), (2.7), whose stability together with that of the outflow part in $(2.4),(2.2 \mathrm{c})$ is equivalent to the overall stability of approximation (2.2). 
Since the $A_{j \sigma}$ and $C_{j \sigma}^{\mathrm{II}}$ are diagonal, we proceed to write

$$
A_{j \sigma}=\operatorname{diag}\left(a_{j \sigma}\right), \quad C_{j \sigma}^{\mathrm{II}}=\operatorname{diag}\left(c_{j \sigma}\right),
$$

and further split (2.4), (2.2c) into $l$ scalar components, each of the form

$$
Q_{-1} v_{\nu}(t+\Delta t)=\sum_{\sigma=0}^{s} Q_{\sigma} v_{\nu}(t-\sigma \Delta t), \quad \nu=r, r+1, \ldots
$$

$$
Q_{\sigma}=\sum_{j=-r}^{p} a_{j \sigma} E^{j}, \quad \sigma=-1, \ldots, s,
$$

$$
\begin{gathered}
v_{\nu}(\mu \Delta t)=\stackrel{\circ}{v}_{\nu}(\mu \Delta t), \quad \mu=0, \ldots, s, \nu=0,1,2, \ldots, \\
T_{-1} v_{\nu}(t+\Delta t)=\sum_{\sigma=0}^{q} T_{\sigma} v_{\nu}(t-\sigma \Delta t), \quad \nu=0, \ldots, r-1, \\
T_{\sigma}=\sum_{j=0}^{m} c_{j \sigma} E^{j}, \quad \sigma=-1, \ldots, q, c_{0(-1)} \neq 0,
\end{gathered}
$$

where the basic scheme $(2.8 \mathrm{a})$ is consistent with a corresponding outflow component of (2.3):

$$
\partial u(x, t) / \partial t=a \partial u(x, t) / \partial x, \quad a=\text { constant }>0 .
$$

Similarly, we split the inflow approximation (2.5), (2.7) into $n-l$ scalar components,

$$
Q_{-1} v_{\nu}(t+\Delta t)=\sum_{\sigma=0}^{s} Q_{\sigma} v_{\nu}(t-\sigma \Delta t), \quad \nu=r, r+1, \ldots
$$

$$
Q_{\sigma}=\sum_{j=-r}^{p} a_{j \sigma} E^{j}, \quad \sigma=-1, \ldots, s
$$

$$
\begin{gathered}
v_{\nu}(\mu \Delta t)=\dot{v}_{\nu}(\mu \Delta t), \quad \mu=0, \ldots, s, \nu=0,1,2, \ldots, \\
v_{\nu}(t+\Delta t)=0, \quad \nu=0, \ldots, r-1,
\end{gathered}
$$

where now (2.10a) is consistent with an inflow component of (2.3):

$$
\partial u(x, t) / \partial t=a \partial u(x, t) / \partial x, \quad a<0 .
$$

Since approximation (2.2) is stable if and only if (2.4), (2.2c) and (2.5), (2.7) are, and since the latter are stable if and only if their scalar components are, we immediately obtain

Lemma 2.2 [3, Lemma 2.2]. Approximation (2.2) is stable if and only if the approximations in (2.8) and (2.10) are stable for all eigenvalues $a>0$ of $A^{\mathrm{I}}$, and $a<0$ of $A^{\mathrm{II}}$, respectively.

With this lemma is mind we proved

Lemma 2.3 [3, Lemma 2.3]. The inflow scalar approximation (2.10) is unconditionally stable for every eigenvalue $a<0$ of $A^{\mathrm{II}}$. 
This lemma-due to Kreiss [7] in the case where the basic scheme is dissipative, explicit and two-level-combined with the previous two, finally yields the main result of this section:

THEOREM 2.1 (compare [4, Theorem 1.1], [3, Theorem 2.1]). Approximation (1.4)-(1.6) is stable if and only if the reduced outflow scalar approximation (2.8) is stable for every eigenvalue $a>0$ of $A^{\mathrm{I}}$. That is, approximation (1.4)-(1.6) is stable if and only if the scalar outflow components of its principal part are all stable.

3. Statement of Main Results and Examples. Theorem 2.1 implies that from now on we may restrict our stability study to the scalar outflow approximation (2.8). Hence, we begin this section by stating the following five assumptions which we impose throughout this paper and which guarantee the validity of the GustafssonKreiss-Sundström theory in [5] for this approximation.

Assumption 3.1 ([4, Assumption 1.1]; [5, Assumption 3.1]). Approximation (2.8) is boundedly solvable; i.e., there exists a constant $K>0$ such that for each $y \in l_{2}(\Delta x)$ there is a unique solution $w \in l_{2}(\Delta x)$ to the equations

$$
Q_{-1} w_{\nu}=y_{\nu}, \quad \nu=r, r+1, \ldots ; \quad T_{-1} w_{\nu}=y_{\nu}, \quad \nu=0, \ldots, r-1,
$$

with $\|w\| \leqslant K\|y\|$, where $Q_{-1}$ and $T_{-1}$ are defined in $(2.8 \mathrm{a}, \mathrm{c})$, and $l_{2}(\Delta x)$ is the space of all grid functions $w=\left\{w_{\nu}\right\}_{\nu=0}^{\infty}$ with $\|w\|^{2} \equiv \Delta x \sum_{\nu=0}^{\infty}\left|w_{\nu}\right|^{2}<\infty$.

Assumption 3.2 ([4, Assumption 1.2]; [5, Assumption 5.1]). The basic scheme (2.8a) is stable for the pure Cauchy problem $-\infty<\nu<\infty$. That is, defining the basic characteristic function

$$
P(z, \kappa) \equiv \sum_{j=-r}^{p} a_{j}(z) \kappa^{j},
$$

where

$$
a_{j}(z) \equiv a_{j(-1)}-\sum_{\sigma=0}^{s} z^{-\sigma-1} a_{j \sigma}, \quad j=-r, \ldots, p
$$

then:

(i) The basic scheme fulfills the von Neumann condition; i.e., the roots $z(\kappa)$ of the basic characteristic equation

$$
P(z, \kappa)=0
$$

satisfy

$$
|z(\kappa)| \leqslant 1 \quad \text { for all } \kappa \text { with }|\kappa|=1 .
$$

(ii) If $|\kappa|=1$, and if $z(\kappa)$ is a root of $P(z, \kappa)$ with $|z(\kappa)|=1$, then $z(\kappa)$ is a simple root of $P(z, \kappa)$.

Assumption 3.3 (compare [4, Assumption 1.3] and [5, Assumption 5.4 together with Definition 10.1]). The basic scheme (2.8a) belongs to one of the following classes:

(i) Dissipative schemes; i.e., schemes for which the roots $z(\kappa)$ of $P(z, \kappa)$ satisfy

$$
|z(\kappa)|<1 \text { for all } \kappa \text { with }|\kappa|=1, \kappa \neq 1 \text {. }
$$

(ii) Almost-dissipative schemes, where the roots of $P(z, \kappa)$ satisfy

$$
|z(\kappa)|<1 \text { for all but a finite number of } \kappa \text { with }|\kappa|=1 \text {, }
$$


(iii) Unitary schemes (also known as strictly nondissipative schemes), where the roots of $P(z, \kappa)$ satisfy

$$
|z(\kappa)|=1 \quad \text { for all } \kappa \text { with }|\kappa|=1 .
$$

Obviously, if the basic scheme belongs to any of these classes, then by continuity it fulfills the von Neumann condition in Assumption 3.2.

Assumption 3.4 ([4, Assumption 1.4]; [5, Assumption 5.5]).

$$
a_{-r}(z), a_{p}(z) \neq 0 \text { for all }|z| \geqslant 1 \text {. }
$$

Assumption 3.5 [4, Assumption 1.5].

$$
\sum_{j=0}^{m}\left|c_{j}(z)\right|>0 \text { for all }|z| \geqslant 1,
$$

where in analogy with (3.2),

$$
c_{j}(z) \equiv c_{j(-1)}-\sum_{\sigma=0}^{q} z^{-\sigma-1} c_{j \sigma}, \quad j=0, \ldots, m .
$$

The above five assumptions are satisfied by all reasonable approximations of the form (2.8).

In order to state our new stability criteria, we define, in complete analogy with (3.1), the boundary characteristic function

$$
R(z, \kappa) \equiv \sum_{j=0}^{m} c_{j}(z) \kappa^{j},
$$

where the $c_{j}(z)$ are given in (3.3). Putting

$$
\Omega(z, \kappa) \equiv|P(z, \kappa)|+|R(z, \kappa)|,
$$

we shall prove in Section 4 the following modification of Theorem 2.1 in [4]:

Theorem 3.1 (1st Main Theorem). Approximation (2.8) is stable if

$$
\begin{aligned}
& \Omega(z, \kappa)>0 \\
& \quad \text { for all }\{|z|=|\kappa|=1,(z, \kappa) \neq(1,1)\} \cup\{|z| \geqslant 1,0<|\kappa|<1\} .
\end{aligned}
$$

This result is an improvement of Theorem 2.1 in [4] in that here, the $(z, \kappa)$ domain on which $\Omega(z, \kappa)$ must not vanish is a proper subset of the corresponding domain in [4].

As in [4], we shall find it convenient to divide this $(z, \kappa)$ domain into three disjoint parts, and restate Theorem 3.1 as follows:

TheOREM 3.1' (Theorem 3.1 restated). Approximation (2.8) is stable if

$$
\begin{array}{ll}
\Omega(z, \kappa)>0 & \text { for all }|z|=|\kappa|=1, \kappa \neq 1, \\
\Omega(z, \kappa=1)>0 & \text { for all }|z|=1, z \neq 1, \\
\Omega(z, \kappa)>0 & \text { for all }|z| \geqslant 1,0<|\kappa|<1 .
\end{array}
$$

The advantage of this setting over that of Theorem 3.1 will be soon clarified by Lemma 3.1, in which we provide helpful sufficient conditions for each of the three inequalities in (3.5) to hold. Before stating this lemma, we need, however, the 
following definitions:

Definition 3.1. The boundary conditions (2.8c) fulfill the von Neumann condition if the roots $z(\kappa)$ of the boundary characteristic function $R(z, \kappa)$ satisfy

$$
|z(\kappa)| \leqslant 1 \quad \text { for all }|\kappa|=1 .
$$

Definition 3.2. The boundary conditions (2.8c) are dissipative if the roots of $R(z, \kappa)$ satisfy

$$
|z(\kappa)|<1 \text { for all }|\kappa|=1, \kappa \neq 1 .
$$

Clearly, these definitions are analogous to those made for the basic scheme in Assumptions 3.2 and 3.3. Further, if the boundary conditions are dissipative, then they obviously fulfill the von Neumann condition.

With the above definitions we can now state:

Lemma 3.1 (1st Main Lemma). (i) Inequality (3.5a) holds if either the basic scheme (2.8a) or the boundary conditions (2.8c) are dissipative.

(ii) Inequality (3.5b) holds if any of the following is satisfied:

(a) The basic scheme is two-level.

(b) The basic scheme is three-level and

$$
\Omega(z=-1, \kappa=1)>0 .
$$

(c) The boundary conditions are two-level and at least zero-order accurate as an approximation to Eq. (2.9).

(d) The boundary conditions are three-level, at least zero-order accurate, and (3.6) is satisfied.

(iii) Inequality (3.5c) holds if the boundary conditions fulfill the von Neumann condition and are either explicit or satisfy

$$
T_{-1}(\kappa) \equiv \sum_{j=0}^{m} c_{j(-1)} \kappa^{j} \neq 0 \quad \text { for all } 0<|\kappa| \leqslant 1 .
$$

This lemma follows immediately from Theorem 2.2 and Lemma 2.1 in [4]. Part (iii) of the lemma is associated with important observations on solvability by Osher [11].

With Theorems 3.1, 3.1' and Lemma 3.1 at hand, it is now a trivial matter to reestablish Examples 2.1-2.10 in [4], precisely as it was carried out using Theorems 2.1, 2.1', 2.2 and Lemma 2.1 there. Thus we conveniently obtain the following five examples:

Example 3.1 [4, Examples 2.1 and 2.2]. Stability holds for any basic scheme of the form ( $2.8 \mathrm{a})$, with boundary conditions generated by either the explicit right-sided, dissipative Euler scheme:

$$
v_{\nu}(t+\Delta t)=v_{\nu}(t)+\lambda a\left[v_{\nu+1}(t)-v_{\nu}(t)\right], \quad 0<\lambda a<1, \nu=0, \ldots, r-1,
$$

or by its implicit analogue:

$$
\begin{aligned}
& v_{\nu}(t+\Delta t)=v_{\nu}(t)+\lambda a\left[v_{\nu+1}(t+\Delta t)-v_{\nu}(t+\Delta t)\right], \\
& \lambda a>0, \nu=0, \ldots, r-1 .
\end{aligned}
$$


Example 3.2 [4, Examples 2.3 and 2.8]. We have stability for any two-level basic scheme of the form (2.8a), with boundary conditions determined by either horizontal extrapolation of order $k-1$ :

$$
v_{\nu}(t+\Delta t)=\sum_{j=1}^{k}\left(\begin{array}{l}
k \\
j
\end{array}\right)(-1)^{j+1} v_{\nu+j}(t+\Delta t), \quad \nu=0, \ldots, r-1,
$$

or by the right-sided, dissipative, three-level Euler scheme:

$$
\begin{aligned}
v_{\nu}(t+\Delta t)=v_{\nu}(t-\Delta t)+2 \lambda a\left[v_{\nu+1}(t-\Delta t)\right. & \left.-v_{\nu}(t-\Delta t)\right] \\
0 & <\lambda a<\frac{1}{2}, \nu=0, \ldots, r-1 .
\end{aligned}
$$

Example 3.3 [4, Examples 2.4 and 2.5]. Stability holds for any dissipative basic scheme of the form (2.8a), with unitary ${ }^{\dagger}$ boundary conditions generated by either oblique extrapolation of order $k-1$ :

$$
v_{\nu}(t+\Delta t)=\sum_{j=1}^{k}\left(\begin{array}{l}
k \\
j
\end{array}\right)(-1)^{j+1} v_{\nu+j}[t-(j-1) \Delta t], \quad \nu=0, \ldots, r-1
$$

or by the second-order accurate Box-scheme:

$$
\begin{aligned}
v_{\nu}(t & +\Delta t)+v_{\nu+1}(t+\Delta t)-\lambda a\left[v_{\nu+1}(t+\Delta t)-v_{\nu}(t+\Delta t)\right] \\
& =v_{\nu}(t)+v_{\nu+1}(t)+\lambda a\left[v_{\nu+1}(t)-v_{\nu}(t)\right], \quad \nu=0, \ldots, r-1 .
\end{aligned}
$$

Example 3.4 [4, Examples 2.6 and 2.7]. We have stability for any basic scheme of the form (2.8a) whose characteristic function satisfies

$$
P(z=-1, \kappa=-1) \neq 0,
$$

with boundary conditions determined by the right-sided three-level, almost-dissipative, weighted Euler scheme:

$$
\begin{aligned}
& v_{\nu}(t+\Delta t)=v_{\nu}(t-\Delta t)+\lambda a\left[2 v_{\nu+1}(t)-v_{\nu}(t+\Delta t)-v_{\nu}(t-\Delta t)\right] \\
& 0<\lambda a<1, \nu=0, \ldots, r-1 .
\end{aligned}
$$

We observe that, if the basic scheme is dissipative, then by Assumption 3.3(i),

$$
P(z, \kappa) \neq 0, \quad|z| \geqslant 1,|\kappa|=1, \kappa \neq 1 ;
$$

so (3.9) holds automatically. As noted already in Example 2.7 of [4], however, certain well-known nondissipative schemes satisfy (3.9) as well. This includes implicit unconditionally stable cases such as the unitary Crank-Nicolson scheme:

$$
\begin{aligned}
v_{\nu}(t & +\Delta t)-\frac{\lambda a}{4}\left[v_{\nu+1}(t+\Delta t)-v_{\nu-1}(t+\Delta t)\right] \\
& =v_{\nu}(t)+\frac{\lambda a}{4}\left[v_{\nu+1}(t)-v_{\nu-1}(t)\right], \quad \nu=r, r+1, \ldots,
\end{aligned}
$$

and the almost-dissipative backward Euler scheme:

$$
\begin{aligned}
v_{\nu}(t+\Delta t)-\frac{\lambda a}{2}\left[v_{\nu+1}(t+\Delta t)-v_{\nu-1}(t+\Delta t)\right]=v_{\nu}(t) & \\
\nu & =r, r+1, \ldots .
\end{aligned}
$$

${ }^{\dagger}$ The boundary conditions are unitary if the roots $z(\kappa)$ of $R(z, \kappa)$ satisfy $|z(\kappa)|=1$ for all $|\kappa|=1$. 
Example 3.5 [4, Examples 2.9 and 2.10]. The Crank-Nicolson scheme in (3.12) with $0<\lambda a \leqslant 1$, and the backward Euler scheme in (3.13), are stable with oblique extrapolation at the boundary:

$$
v_{0}(t+\Delta t)=\sum_{j=1}^{k}\left(\begin{array}{l}
k \\
j
\end{array}\right)(-1)^{j+1} v_{j}[t-(j-1) \Delta t] .
$$

While Examples 3.1-3.5 include and extend many of the cases discussed in the recent literature (e.g., [1]-[8], [10], [12]-[15]), other interesting examples are not covered by Theorems 3.1 and 3.1'. For instance, Gustafsson et al. showed in Theorem 6.2 of [5] that the Leap-Frog scheme

$$
\begin{aligned}
v_{\nu}(t+\Delta t)=v(t-\Delta t)+\lambda a\left[v_{\nu+1}(t)-v_{\nu-1}(t)\right], \\
0<\lambda a<1, \nu=1,2,3, \ldots,
\end{aligned}
$$

with the oblique boundary extrapolation in (3.14) provides a stable approximation. In this case, however, the characteristic functions are

$$
P(z, \kappa)=1-z^{-2}-\lambda a z^{-1}\left(\kappa-\kappa^{-1}\right)
$$

and

$$
R(z, \kappa)=1-\sum_{j=1}^{k}\left(\begin{array}{l}
k \\
j
\end{array}\right)(-1)^{j+1} z^{-j} \kappa^{j}=\left(1-z^{-1} \kappa\right)^{k}
$$

Therefore, $\Omega(z, \kappa)>0$ at all points $(z, \kappa)$ in (3.4), except for

$$
\Omega(z=-1, \kappa=-1)=|P(z=-1, \kappa=-1)|+|R(z=-1, \kappa=-1)|=0 ;
$$

so (3.4) fails, and Theorem 3.1 does not imply stability.

This counterexample-which shows that our criteria in Theorems 3.1 and 3.1' are sufficient but not necessary for stability - is not the only one. As we shall see, there are other well-known approximations for which $\Omega(z, \kappa)$ does not vanish on the $(z, \kappa)$ domain in (3.4), with the exception of the particular point $(z, \kappa)=(-1,-1)$. With this in mind, we shall prove in Section 4 the following alternative to Theorem 3.1, where the point $(z, \kappa)=(-1,-1)$ is treated separately.

TheOREM 3.2 (2nd Main Theorem). Approximation (2.8) is stable if

$$
\frac{\partial P(z, \kappa)}{\partial z} /\left.\frac{\partial P(z, \kappa)}{\partial \kappa}\right|_{z=\kappa=-1}<0
$$

and

$$
\begin{aligned}
& \Omega(z, \kappa)>0 \\
& \quad \text { for all }\{|z|=|\kappa|=1,(z, \kappa) \neq \pm(1,1)\} \cup\{|z| \geqslant 1,0<|\kappa|<1\} .
\end{aligned}
$$

Having Theorem 3.2, we follow the idea that led to Theorem 3.1', and split the $(z, \kappa)$ domain in (3.19) into three disjoint sets in order to obtain:

TheOREM 3.2' (Theorem 3.2 restated). Approximation (2.8) is stable if (3.18) holds and

(3.20b) $\quad \Omega(z, \kappa=1)>0$ for all $|z|=1, z \neq 1$,

(3.20c) $\quad \Omega(z, \kappa)>0 \quad$ for all $|z| \geqslant 1,0<|\kappa|<1$. 
Since the inequalities in $(3.20 \mathrm{~b}, \mathrm{c})$ coincide with those in $(3.5 \mathrm{~b}, \mathrm{c})$, then Lemma 3.1 immediately yields:

LEMMA 3.2 (2nd Main Lemma). Inequalities (3.20b) and (3.20c) hold under the hypotheses in parts (ii) and (iii) of Lemma 3.1, respectively.

We deliberately choose not to apply part (i) of Lemma 3.1 to the inequality in (3.20a), since if the basic scheme or the boundary conditions are dissipative, then by Assumption 3.3(i) and Definition 3.2, we have either (3.11) or

$$
R(z, \kappa) \neq 0, \quad|z| \geqslant 1,|\kappa|=1, \kappa \neq 1 \text {. }
$$

So

$$
\Omega(z=-1, \kappa=-1)>0 ;
$$

and Theorems 3.2 and $3.2^{\prime}$ lose any possible advantage they may have had over Theorems 3.1 and $3.1^{\prime}$.

Example 3.6. Consider any basic scheme of the form (2.8a) whose characteristic function satisfies (3.18) as well as

$$
P(z, \kappa) \neq 0, \quad z=\kappa,|\kappa|=1, \kappa \neq \pm 1 ;
$$

and determine the boundary conditions by oblique extrapolation as in (3.7). The boundary characteristic function, given in (3.17), satisfies

$$
\Omega(z, \kappa) \geqslant|R(z, \kappa)|>0, \quad z \neq \kappa .
$$

In addition, by (3.21),

$$
\Omega(z, \kappa) \geqslant|P(z, \kappa)|>0, \quad z=\kappa,|\kappa|=1, \kappa \neq \pm 1 .
$$

So (3.22) and (3.23) imply (3.19), and Theorem 3.2 yields stability.

The inequalities in (3.18) and (3.21) are met by a number of well-known schemes, including:

(a) The unitary Leap-Frog scheme in (3.15) (compare [5, Theorem 6.2]), whose characteristic function in (3.16) satisfies

$$
\frac{\partial P(z, \kappa)}{\partial z} /\left.\frac{\partial P(z, \kappa)}{\partial \kappa}\right|_{z=\kappa=-1}=\frac{-1}{\lambda a}<0
$$

and

$$
P(z, \kappa)=(1-\lambda a)\left(1-\kappa^{-2}\right) \neq 0, \quad z=\kappa,|\kappa|=1, \kappa \neq \pm 1(0<\lambda a<1) .
$$

(b) The unitary, five-point Leap-Frog scheme (e.g., [8], [9]):

$$
\begin{aligned}
v_{\nu}(t+\Delta t)= & v_{\nu}(t-\Delta t)+\frac{4 \lambda a}{3}\left[v_{\nu+1}(t)-v_{\nu-1}(t)\right] \\
& -\frac{\lambda a}{6}\left[v_{\nu+2}(t)-v_{\nu-2}(t)\right], \quad 0<\lambda a<\frac{3}{5}, \nu=2,3, \ldots
\end{aligned}
$$

Here,

$$
P(z, \kappa)=1-z^{-2}+\frac{1}{6} \lambda a z^{-1}\left(\kappa-\kappa^{-1}\right)\left(\kappa+\kappa^{-1}-8\right)
$$

so

$$
\frac{\partial P(z, \kappa)}{\partial z} /\left.\frac{\partial P(z, \kappa)}{\partial \kappa}\right|_{z=\kappa=-1}=\frac{-3}{5 \lambda a}<0
$$

and

$$
P(z, \kappa)=\left(1-\kappa^{-2}\right)\left[1+\frac{1}{6} \lambda a\left(\kappa+\kappa^{-1}-8\right)\right] \neq 0, \quad z=\kappa,|\kappa|=1, \kappa \neq \pm 1 .
$$


(c) The almost-dissipative Lax-Friedrichs scheme (e.g., [10]):

$$
\begin{aligned}
v_{\nu}(t+\Delta t)=\frac{1}{2}\left[v_{\nu+1}(t)+v_{\nu-1}(t)\right]+\frac{\lambda a}{2}\left[v_{\nu+1}(t)-v_{\nu-1}(t)\right] \\
0<\lambda a<1, \nu=1,2,3, \ldots
\end{aligned}
$$

for which

$$
P(z, \kappa)=1-\frac{z^{-1}}{2}\left[\kappa+\kappa^{-1}+\lambda a\left(\kappa-\kappa^{-1}\right)\right]
$$

so again,

$$
\frac{\partial P(z, \kappa)}{\partial z} /\left.\frac{\partial P(z, \kappa)}{\partial \kappa}\right|_{z=\kappa=-1}=\frac{-1}{\lambda a}<0
$$

and

$$
P(z, \kappa)=\frac{1}{2}(1-\lambda a)\left(1-\kappa^{-2}\right) \neq 0, \quad z=\kappa,|\kappa|=1, \kappa \neq \pm 1 .
$$

We note that the stability question for these three schemes, combined with oblique extrapolation at the boundary, could not have been handled by Theorem 3.1 since here,

$$
\Omega(z=-1, \kappa=-1)=0 .
$$

Example 3.7 (compare [4, Example 2.7]). Take any basic scheme that satisfies (3.18), and let the boundary conditions be determined by the first-order accurate, weighted Euler scheme in (3.10). The boundary characteristic function is

$$
R(z, \kappa)=1-z^{-2}-\lambda a\left(2 \kappa z^{-1}-1-z^{-2}\right) ;
$$

so its roots $z(\kappa)$ satisfy

$$
z\left(\kappa=e^{i \xi}\right)=e^{i \xi} \frac{\lambda a \pm \Phi(\xi)}{\lambda a+1}, \quad \Phi(\xi) \equiv \sqrt{(\lambda a)^{2}+e^{-2 i \xi}\left[1-(\lambda a)^{2}\right]}
$$

Denoting $\rho=(\lambda a)^{2}$, we obtain

$$
\begin{array}{r}
|\Phi(\xi)|^{4}=\rho^{2}+(1-\rho)^{2}+2 \rho(1-\rho) \cos 2 \xi<\rho^{2}+(1-\rho)^{2}+2 \rho(1-\rho)=1 \\
0<|\xi|<\pi(0<\rho<1)
\end{array}
$$

hence by (3.28),

$$
\left|z\left(\kappa=e^{i \xi}\right)\right| \leqslant \frac{\lambda a+|\Phi(\xi)|}{\lambda a+1}<1, \quad 0<|\xi|<\pi .
$$

Consequently,

$$
R(z, \kappa) \neq 0, \quad|z| \geqslant 1,|\kappa|=1, \kappa \neq \pm 1
$$

By (3.28) again,

$$
z(\kappa=-1)=-\frac{\lambda a \pm 1}{\lambda a+1}
$$

hence one root is $z(\kappa=-1)=-1$ and the other satisfies $|z(\kappa=-1)|<1$; so

$$
R(z, \kappa=-1) \neq 0, \quad|z| \geqslant 1, z \neq-1 .
$$

Collecting (3.30), (3.31) we find, therefore, that

$$
\Omega(z, \kappa) \geqslant|R(z, \kappa)|>0, \quad|z| \geqslant 1,|\kappa|=1, \kappa \neq 1,(z, \kappa) \neq(-1,-1),
$$

and (3.20a) follows. 
Next, since

$$
\Omega(z=-1, \kappa=1) \geqslant|R(z=-1, \kappa=1)|=4 \lambda a>0,
$$

then Lemmas 3.1(ii)(d) and 3.2 imply (3.20b).

Further, by (3.29), the roots of $R(z, \kappa)$ satisfy

$$
\left|z\left(\kappa=e^{i \xi}\right)\right| \leqslant 1, \quad|\xi| \leqslant \pi
$$

so our boundary conditions fulfill the von Neumann condition. Moreover, we easily verify that

$$
T_{-1}(\kappa)=1-\lambda a \kappa \neq 0, \quad 0<|\kappa| \leqslant 1(0<\lambda a<1) .
$$

Hence, Lemmas 3.1(iii) and 3.2 assure (3.20c), and by Theorem $3.2^{\prime}$ we have stability.

We recall that the Leap-Frog scheme in (3.15), the five-point Leap-Frog scheme in (3.24), and the Lax-Friedrichs schemes in (3.25), all satisfy (3.18); so they fit into the above example.

We also point out that the stable approximation (3.25), (3.10) was mistakenly declared unstable in [4].

Example 3.8. Consider the almost-dissipative Lax-Friedrichs scheme in (3.25), with the Box-scheme boundary conditions in (3.8). As indicated in (3.27), the characteristic function in (3.26) fulfills (3.18). Also, the roots of $P(z, \kappa)$ satisfy

$$
z\left(\kappa=e^{i \xi}\right)=\cos \xi+i \lambda a \sin \xi
$$

so

$$
z(\kappa=-1)=-1
$$

and

$$
\left|z\left(\kappa=e^{i \xi}\right)\right|^{2}=\cos ^{2} \xi+(\lambda a)^{2} \sin ^{2} \xi<1, \quad 0<|\xi|<\pi(0<\lambda a<1) .
$$

Thus,

$$
P(z, \kappa) \neq 0, \quad|z| \geqslant 1,|\kappa|=1, \kappa \neq 1,(z, \kappa) \neq(-1,-1)
$$

hence

$$
\Omega(z, \kappa)>|P(z, \kappa)|>0, \quad|z| \geqslant 1,|\kappa|=1, \kappa \neq 1,(z, \kappa) \neq(-1,-1),
$$

and we obtain (3.20a).

In addition, Lemmas 3.1(ii)(c) and 3.2 immediately give (3.20b). Also, by (3.30) we get

$$
\left|z\left(\kappa=e^{i \xi}\right)\right| \leqslant 1, \quad|\xi| \leqslant \pi
$$

so the boundary conditions fulfill the von Neumann condition. And since

$$
\operatorname{Re} T_{-1}(\kappa)=1+\operatorname{Re}(\kappa)+\lambda a[1-\operatorname{Re}(\kappa)] \neq 0, \quad|\kappa| \leqslant 1(0<\lambda a<1),
$$

then Lemmas 3.1(iii) and 3.2 yield (3.20c), so Theorem 3.2' implies stability.

4. Proof of Main Results. In [4] we proved

TheOREM 4.1 [4, Theorem 2.1]. Approximation (2.8) is stable if

$$
\Omega(z, \kappa)>0 \text { for }|z| \geqslant 1,0<|\kappa| \leqslant 1,(z, \kappa) \neq(1,1) .
$$


From this we can easily obtain the following

Proof of Theorem 3.1. Since by Assumption 3.2 the basic scheme fulfills the von Neumann condition, then the characteristic function in (3.1) satisfies

$$
P(z, \kappa) \neq 0 \text { for }|z|>1,|\kappa|=1 .
$$

Thus, we always have

$$
\Omega(z, \kappa)>0, \quad|z|>1,|\kappa|=1,
$$

which, together with the statement of Theorem 4.1, completes the proof.

In order to prove Theorem 3.2, we again consider $P(z, \kappa)$. By Assumption 3.4, for each $z$ with $|z| \geqslant 1, P(z, \kappa)$ has $r+p$ roots $\kappa(z)$. These continuous roots, which play a major role in the stability analysis of Approximation (2.8), have the following separation property.

LEMmA 4.1 ([4, Lemma 3.1]; compare [5, Lemmas 5.1 and 5.2]). For $|z|>1$, the characteristic function $P(z, \kappa)$ has precisely $r$ roots $\kappa(z)$ with $0<|\kappa(z)|<1$, p roots with $|\kappa(z)|>1$, and no roots with $|\kappa(z)|=1$.

By this lemma, the roots $\kappa(z)$ of $P(z, \kappa)$ split for $|z|>1$ into two groups: $r$ inner roots satisfying $|\kappa(z)|<1$, and $p$ outer roots with $|\kappa(z)|>1$. Using a continuity argument, we observe that these groups of inner and outer roots remain well-defined for $|z| \geqslant 1$ as well, where milder inequalities, $|\kappa(z)| \leqslant 1$ and $|\kappa(z)| \geqslant 1$, hold, respectively. Here, of course, if for $z_{0}$ with $\left|z_{0}\right|=1$ we have $\left|\kappa\left(z_{0}\right)\right|=1$, then $\kappa\left(z_{0}\right)$ is an inner (outer) root of $P\left(z_{0}, \kappa\right)$ if and only if, as $z,|z|>1$, approaches $z_{0}$, there exists an inner (outer) root $\kappa(z)$ of $P(z, \kappa)$ that satisfies $\kappa(z) \rightarrow \kappa\left(z_{0}\right)$. Since by Assumption 3.4, $\kappa=0$ is not a root of $P(z, \kappa)$ for $|z| \geqslant 1$, we summarize the above argument as follows:

Lemma 4.2 [4, Lemma 3.2]. For $|z| \geqslant 1$, the $r+p$ roots $\kappa(z)$ of the basic characteristic function $P(z, \kappa)$ split into $r$ inner roots with $0<|\kappa(z)| \leqslant 1$ and $p$ outer roots with $|\kappa(z)| \geqslant 1$.

We can quote now the following stability criterion:

THEOREM 4.2 [4, Corollary 3.1]. Approximation (2.8) is stable if and only if for every $z$ with $|z| \geqslant 1$ and each corresponding inner root $\kappa(z)$ of $P(z, \kappa)$,

$$
\Omega(z, \kappa(z)) \equiv|P(z, \kappa(z))|+|R(z, \kappa(z))|>0 .
$$

We shall also need

LEMMA 4.3. (i) $\kappa=1$ is not an inner root of $P(z, \kappa)$ for $z=1$.

(ii) If (3.18) holds, then $\kappa=-1$ is not an inner root for $z=-1$.

Proof. (i) The first part of the lemma is precisely the content of Lemma 3.3 in [4].

(ii) If $P(z=-1, \kappa=-1) \neq 0$, then there is nothing to prove, so suppose that

$$
P(z=-1, \kappa=-1)=0 \text {. }
$$


By (3.18),

$$
\left.\frac{\partial P(z, \kappa)}{\partial \kappa}\right|_{z=\kappa=-1} \neq 0
$$

thus, having (4.2) and (4.3), we employ the implicit function theorem and find that in some neighborhood $\mathbf{N}$ of $z=-1$, the characteristic equation

$$
P(z, \kappa)=0
$$

has a unique differentiable solution $\kappa=\hat{\kappa}(z)$ satisfying

$$
\hat{\kappa}(z=-1)=-1 \text {. }
$$

Since

$$
P(z, \hat{\kappa}(z)) \equiv 0 \quad \text { for } z \in \mathbf{N}
$$

then

$$
\frac{\partial P(z, \kappa)}{\partial z}+\left.\frac{\partial P(z, \kappa)}{\partial \kappa} \frac{d \hat{\kappa}(z)}{d z}\right|_{z=\kappa=-1}=0 .
$$

Hence, (3.18) and (4.5) imply

$$
\left.\frac{d \hat{\kappa}(z)}{d z}\right|_{z=-1}=-\frac{\partial P(z, \kappa)}{\partial z} /\left.\frac{\partial P(z, \kappa)}{\partial \kappa}\right|_{z=\kappa=-1} \equiv \gamma>0 .
$$

By (4.4) and (4.6), therefore, for $z=-1-\varepsilon$ with sufficiently small $\varepsilon>0$, a Taylor expansion gives

$$
\begin{aligned}
\hat{\kappa}(z=-1-\varepsilon) & =\hat{\kappa}(z=-1)-\left.\varepsilon \frac{d \hat{\kappa}(z)}{d z}\right|_{z=-1}+O\left(\varepsilon^{2}\right) \\
& =-1-\gamma \varepsilon+O\left(\varepsilon^{2}\right)<-1 .
\end{aligned}
$$

Thus, for $z$ in some left real neighborhood of -1 we have

$$
|\hat{\kappa}(z)|>1 \text {. }
$$

Since $\hat{\kappa}(z)$ is a continuous function in $\mathbf{N}$, then by Lemma 4.1 the inequality in (4.7) holds for all $z \in \mathbf{N},|z|>1$. That is, $\hat{\kappa}(z)$ is an outer root of $P(z, \kappa)$ for $z \in \mathbf{N}$, $|z|>1$. Since in addition,

$$
\hat{\kappa}(z) \underset{z \rightarrow-1}{\rightarrow \rightarrow}-1
$$

then by definition, $\kappa=-1$ is an outer root for $z=-1$, and the proof is complete.

Proof of Theorem 3.2. By (3.18) and Lemma 4.3, $\kappa=1$ and $\kappa=-1$ are excluded as inner roots of $P(z, \kappa)$ for $z=1$ and $z=-1$, respectively. Thus, in view of Theorem 4.2 and Lemma 4.2, approximation (2.8) is stable if

$$
\Omega(z, \kappa)>0 \text { for all }|z| \geqslant 1,0<|\kappa| \leqslant 1,(z, \kappa) \neq \pm(1,1) .
$$

Since the basic scheme fulfills the von Neumann condition, then, as shown in the proof of Lemma 4.1, we automatically have (4.1). But (4.1) combined with the hypothesis in (3.19) implies (4.8), and stability follows. 
Department of Mathematics

Technion-Israel Institute of Technology

Haifa 32000, Israel

School of Mathematical Sciences

Tel Aviv University

Tel Aviv 69978, Israel

1. M. Goldberg, “On a boundary extrapolation theorem by Kreiss,” Math. Comp., v. 31, 1977, pp. 469-477.

2. M. GOLDBERG \& E. TADMOR, "Scheme-independent stability criteria for difference approximations of hyperbolic initial-boundary value problems. I," Math. Comp., v. 32, 1978, pp. 1097-1107.

3. M. GOLDBERG \& E. TADMOR, "Scheme-independent stability criteria for difference approximations of hyperbolic initial-boundary value problems. II," Math. Comp., v. 36, 1981, pp. 603-626.

4. M. Goldberg \& E. TADMOR, "Convenient stability criteria for difference approximations of hyperbolic initial-boundary value problems," Math. Comp., v. 44, 1985, pp. 361-377.

5. B. GustafsSon, H.-O. KREISS \& A. SUnDSTROM, "Stability theory of difference approximations for mixed initial boundary value problems. II,” Math. Comp., v. 26, 1972, pp. 649-686.

6. B. GuSTAFSSON \& J. OLIGER, "Stable boundary approximations for implicit time discretizations for gas dynamics,” SIAM J. Sci. Statist. Comput., v. 3, 1982, pp. 408-421.

7. H.-O. KREISS, "Difference approximations for hyperbolic differential equations," in Numerical Solutions of Partial Differential Equations (J. H. Bramble, ed.), Academic Press, New York, 1966, pp. $51-58$.

8. H.-O. Kreiss \& J. Oliger, Methods for the Approximate Solution of Time Dependent Problems, GARP Publication Series No. 10, Geneva, 1973.

9. J. Oliger, "Fourth order difference methods for the initial boundary-value problem for hyperbolic equations,” Math. Comp., v. 28, 1974, pp. 15-25.

10. S. OSHER, "Systems of difference equations with general homogeneous boundary conditions," Trans. Amer. Math. Soc., v. 137, 1969, pp. 177-201.

11. S. OSHER, "Stability of parabolic difference approximations to certain mixed initial-boundary value problems," Math. Comp., v. 26, 1972, pp. 13-39.

12. G. SKOllermo, How the Boundary Conditions Affect the Stability and Accuracy of Some Methods for Hyperbolic Equations, Report No. 62, Dept. of Computer Science, Uppsala University, Uppsala, Sweden, 1975.

13. G. SKöllermo, Error Analysis for the Mixed Initial Boundary Value Problem for Hyperbolic Equations, Report No. 63, Dept. of Computer Science, Uppsala University, Uppsala, Sweden, 1975.

14. E. TADMOR, Scheme-Independent Stability Criteria for Difference Approximations to Hyperbolic Initial-Boundary Value Systems, Ph.D. thesis, Dept. of Mathematical Sciences, Tel Aviv University, Tel Aviv, Israel, 1978.

15. L. N. Trefethen, Wave Propagation and Stability for Finite Difference Schemes, Ph.D. thesis, Report No. STAN-CS-82-905, Computer Science Department, Stanford University, Stanford, California, 1982. 